\title{
Review of clinical trials in intraoperative molecular imaging during cancer surgery
}

John Y. K. Lee

Steve S. Cho

Walter Stummer

Janos L. Tanyi

Alexander L. Vahrmeijer

Eben Rosenthal

Barbara Smith

Eric Henderson

David W. Roberts

Amy Lee

Constantinos G. Hadjipanayis

Jeffrey N. Bruce

Jason G. Newman

Sunil Singhal 


\title{
Review of clinical trials in intraoperative molecular imaging during cancer surgery
}

\author{
John Y. K. Lee, ${ }^{\text {a, }}$ Steve S. Cho, ${ }^{\text {a }}$ Walter Stummer, ${ }^{b}$ Janos L. Tanyi,, ${ }^{a}$ Alexander L. Vahrmeijer, ${ }^{c}$ \\ Eben Rosenthal, ${ }^{d}$ Barbara Smith, ${ }^{e}$ Eric Henderson, ${ }^{\mathrm{f}, \mathrm{g}}$ David W. Roberts, ${ }^{\mathrm{f}, \mathrm{g}}$ Amy Lee, ${ }^{\mathrm{h}}$ \\ Constantinos G. Hadjipanayis, ${ }^{i}$ Jeffrey N. Bruce, ${ }^{j}$ Jason G. Newman, ${ }^{a}$ and Sunil Singhal ${ }^{\mathrm{a}}$ \\ aUniversity of Pennsylvania, Perelman School of Medicine, Philadelphia, Pennsylvania, United States \\ bUniversity of Münster, Münster, Germany \\ 'Leiden University, Medical Center, Leiden, The Netherlands \\ dStanford University, School of Medicine, California, United States \\ eHarvard University, School of Medicine, Boston, Massachusetts, United States \\ fDartmouth College, School of Medicine, Hanover, New Hampshire, United States \\ 'Dartmouth College, School of Engineering, Hanover, New Hampshire, United States \\ hUniversity of Washington, School of Medicine, Seattle, Washington, United States \\ Icahn School of Medicine at Mount Sinai, New York, United States \\ 'Columbia University, School of Medicine, New York, United States
}

\begin{abstract}
Most solid cancers are treated by surgical resections to reduce the burden of disease. Surgeons often face the challenge of detecting small areas of residual neoplasm after resection or finding small primary tumors for the initial resection. Intraoperative molecular imaging (IMI) is an emerging technology with the potential to dramatically improve cancer surgery operations by allowing surgeons to better visualize areas of neoplasm using fluorescence imaging. Over the last two years, two molecular optical contrast agents received U.S. Food and Drug Administration approval, and several more drugs are now on the horizon. Thus a conference was organized at the University of Pennsylvania to bring together oncologic surgeons from different specialties to discuss the current clinical status of IMI trials with a specific focus on phase 2 and phase 3 studies. In addition, phase 1 and experimental trials were also discussed briefly, to highlight other novel techniques. Our review summarizes the discussions from the conference and delves into the types of cancers discussed, different contrast agents in human trials, and the clinical value being studied. ( ) The Authors. Published by SPIE under a Creative Commons Attribution 4.0 Unported License. Distribution or reproduction of this work in whole or in part requires full attribution of the original publication, including its DOI. [DOI: 10.1117/1.JBO.24.12.120901]

Keywords: fluorescence-guided surgery; tumor surgery; intraoperative visualization; molecular imaging

Paper 190282VR received Aug. 27, 2019; accepted for publication Nov. 15, 2019; published online Dec. 5, 2019.
\end{abstract}

\section{Introduction}

The goal of a successful cancer operation is localization and accurate delineation of malignant and benign tissue to maximize removal of cancer, while minimizing removal of noncancerous tissue. Cancer surgeons in a wide range of specialties perform a variety of cancer operations, and each cancer type presents unique challenges in achieving this goal. Common challenges include: (a) identification of small primary, synchronous, or metachronous tumor deposits; (b) accurate selection of lymph nodes with metastatic disease; (c) assessment of tumor margins and differentiation of tumor from surrounding critical structures such as nerves; and (d) recognition of residual disease after tumor cytoreduction.

Traditionally, cancer surgeons have only had two intraoperative tools, visual inspection and manual palpation, to meet these challenges. Intraoperative molecular imaging (IMI) is an emerging technology with the potential to dramatically improve oncologic operations. This technology requires: (a) a fluorescent agent that will selectively accumulate in tumor tissues and (b) a specialized imaging system to detect and quantify the fluorescence. Several features of IMI make it more attractive than other

*Address all correspondence to John Y. K. Lee, E-mail: John.lee3@ pennmedicine.upenn.edu intraoperative adjuncts to a surgeon's hands, eyes, and clinical decision making. First, it does not involve exposure to ionizing radiation. Second, imaging is easy to interpret for surgeons unfamiliar with the technology. Third, IMI causes minimal interruption and integrates well into the normal flow of an operation.

With the recent approval of 5-aminolevulinic acid (5-ALA) and hexaminolevulinate for intraoperative imaging, several other optical contrast agents have emerged for IMI. Clinical trials spanning pilot studies to phase 3 trials are ongoing. Thus on November 2, 2018, several clinical investigators met at the first Annual Intraoperative Molecular Imaging Precision Surgery Meeting at the Perelman School of Medicine of the University of Pennsylvania to discuss the current status of clinical trials in IMI in cancer surgery. This meeting was focused on current phase 2 and phase 3 clinical trials in the United States and Europe, though phase 1 and pilot studies were also discussed. A major goal of this meeting was to determine if IMI was leading to clinical value and what endpoints have been established in clinical trials for different cancer types. The discussions that emerged from the conference proceedings are reviewed in this article (Tables 1 and 2).

\section{FDA-Approved IMI Agents}

Several talks were scheduled for learning about 5-ALA due to the recent U.S. Food and Drug Administration (FDA) approval of this drug for glioma surgery. 
Table 1 Summary of conference proceedings.

\begin{tabular}{|c|c|c|c|}
\hline Tumor type & Contrast agent & Status & Lead discussant \\
\hline Breast cancer & LUM015 & Phase 2 & Barbara Smith \\
\hline Colorectal cancer & SGM-101 & Phase 3 & Alexander Vahrmeijer \\
\hline \multirow[t]{6}{*}{ Glioma } & 5-ALA & FDA approved & Walter Stummer \\
\hline & 5-ALA & FDA approved & Constantinos Hadjipanayis \\
\hline & 5-ALA & FDA approved & David Roberts \\
\hline & TumorGlow (SWIG) & Exploratory & John Y. K. Lee \\
\hline & Fluorescein & Exploratory & Jeffrey Bruce \\
\hline & TumorPaint & Exploratory & Amy Lee \\
\hline \multirow[t]{2}{*}{ Head and neck cancer } & Anti-EGFR antibody & Exploratory & Eben Rosenthal \\
\hline & TumorGlow (SWIG) & Exploratory & Jason Newman \\
\hline Lung cancer & OTL38 & Phase 2 & Sunil Singhal \\
\hline Ovarian cancer & OTL38 & Phase 3 & Janos Tanyi \\
\hline Osteosarcoma & Anti-EGFR affibody & Exploratory phase 0 & Eric Henderson \\
\hline
\end{tabular}

Note: 5-ALA, 5 aminolevulinic acid; EGFR, epidermal growth factor receptor; and ICG, indocyanine green

Table 2 Common indications for IMI for cancer surgeons.

\begin{tabular}{ll}
\hline Common indications & \multicolumn{1}{c}{ Examples } \\
\hline Margin assessment & $\begin{array}{l}\text { Head and neck cancer, } \\
\text { breast cancer, lung cancer, } \\
\text { and brain cancer }\end{array}$ \\
Lymph node status & Head and neck cancer \\
Localization of tumor & $\begin{array}{l}\text { Lung cancer, ductal carcinoma } \\
\text { in situ, and colorectal cancer }\end{array}$ \\
Optical biopsy & $\begin{array}{l}\text { Colorectal cancer, lung cancer, } \\
\text { and brain cancer }\end{array}$ \\
$\begin{array}{ll}\text { Identification of residual disease } \\
\text { after cytoreductive surgery }\end{array}$ & $\begin{array}{l}\text { Ovarian cancer, mesothelioma, } \\
\text { and brain cancer }\end{array}$ \\
\hline
\end{tabular}

\subsection{5-ALA European Experience}

The conference began with Dr. Walter Stummer discussing the European approval and more recent U.S. FDA approval of 5-ALA for high-grade glioma (HGG) tumors. Although the history of intraoperative imaging in neurosurgery includes preoperative MRI, intraoperative MRI, and intraoperative ultrasound, Stummer recognized the deficiencies of these intraoperative techniques as early as 1990. Focusing in on 5-ALA, a prodrug requiring enzymatic conversion in the mitochondria as part of a ubiquitous heme biosynthesis pathway, Stummer started with in vitro and rodent investigations in 1994 . He then progressed to phase $1 / 2$ dose escalation studies in 1998 which then led to the successful European phase 3 clinical trial published in 2006. ${ }^{1}$ In this pivotal study of more than 400 enrolled patients, progression-free survival and gross total resection rate were significantly higher in the 5-ALA group compared to controls. Post hoc analysis also showed that 5-ALA leads to less need for repeat surgeries. 5-ALA was thus approved in Europe and many Asian countries, and European and Asian neurosurgeons have had a decade-long advantage over American colleagues in the incorporation of IMI into routine neurosurgical tumor debulking.

Next, Stummer discussed the challenges in interpretation of 5-ALA test characteristics-sensitivity, specificity, positive predictive value (PPV), and negative predictive value. In HGG surgery, the acquisition of biopsy samples is not performed in a random manner, and thus biases and confounders are introduced into the acquisition of test data. This bias limits the ability to interpret test characteristics. In addition to bias, even the gold standard of H\&E staining does not demonstrate the full neoplastic potential of biopsy specimens, and thus the gold standard definition of true positive margins is not always reproducible.

Dr. Walter Stummer and Dr. Constantinos Hadjipanayis next discussed the myriad challenges of FDA approval for 5-ALA in the United States. In contrast to European regulatory agencies, the U.S. FDA would not accept 5-ALA as a therapeutic drug. Ultimately, the FDA approved 5-ALA as an imaging adjunct for the visualization of malignant tissue during HGG surgery. One of the primary considerations for clinical trial design is the requirement for a "clinically meaningful" endpoint. Given that 5-ALA was not to be considered a therapeutic drug in the United States, conventional endpoints such as overall survival or progression-free survival were not chosen as study endpoints. Instead, a clinically meaningful endpoint was needed to demonstrate "benefit to the patient" and a favorable risk/benefit ratio. In the case of HGG surgery, simple correlations of fluorescence with MRI-location were not enough to imply benefit. Instead, because there was retrospective data that associated greater surgical resection with survival benefit and a reduced need for 
reoperation, the benefit of detecting additional tumor disease with 5-ALA could be implied and was accepted by the FDA in lieu of a formal trial.

Drs. Stummer and Hadjipanayis, in addition to industry partners, NX Development Corporation (NXDC) and photonamic $\mathrm{GmbH}$ and Co., were able to demonstrate to the FDA that PPV of $\geq 97 \%$ was the most important test characteristic for surgeons during the resection of HGGs. Indeed, pooled data presented to FDA for 5-ALA from two European studies in primary HGGs demonstrated sensitivity, specificity, PPV, and negative predictive value (NPV) of $68 \%, 80 \%, 97 \%$, and $21 \%$, respectively. Hence, modest sensitivity and specificity test characteristics were not an impediment to FDA approval of a neurosurgical fluorescent agent for HGG resection, if the agent could be shown to be safe, to have a high PPV, and to provide clinically meaningful improvement in surgical resection of gliomas. Indeed, FDA approval of 5-ALA provided a road map for all the novel fluorophores that were discussed in the remainder of the conference.

\section{$2.25-A L A$ Mount Sinai Experience}

Hadjipanayis and Stummer ${ }^{2}$ described in more detail the journey required in the United States to achieve FDA approval of 5-ALA. In 2011, Dr. Hadjipanayis obtained the first IND for 5-ALA (Gliolan; photonamic GmbH and Co.). He completed a 5-ALA phase 2 study at Emory University where he studied volumetric outcomes of gadolinium-enhancing tumor in HGG patients with a semiautomated software program ${ }^{3,4}$ and wholebrain magnetic spectroscopic imaging. ${ }^{5} \mathrm{He}$ is currently the sponsor of a multicenter 5-ALA US trial in conjunction with the PI, Dr. Bob Carter from Massachusetts General Hospital. Dr. Hadjipanayis discussed the challenges of obtaining 5-ALA FDA approval in the United States since it was initially considered a therapeutic agent. Multiple visits were made to the FDA by Dr. Hadjipanayis, Dr. Stummer, and colleagues in addition to industry partners NXDC and photonamic gmbH. Establishing 5-ALA as an intraoperative diagnostic imaging agent in HGG tumors permitted the acceleration of the US approval after orphan drug designation by the FDA in 2013. Utilization of pivotal European histology-based controlled studies and other data resulted in an FDA new drug application in 2016. An FDA Advisory Committee meeting was conducted on May 10, 2017 resulting in unanimous approval of 5-ALA (Gleolan; NXDC.). The official FDA approval of 5-ALA for the intraoperative visualization of suspected HGGs (WHO grade III or IV) quickly followed on June 6, 2017.

Hadjipanayis et al. ${ }^{6}$ then discussed the role of 5-ALA in other tumor types. For example, Widhalm et al. ${ }^{7}$ have published the use of 5-ALA to detect anaplastic foci (higher-grade portion) present in nonenhancing, presumed low-grade gliomas. 5-ALA has also been studied in meningiomas, as $80 \%$ of these tumors (WHO grades I, II, and III) will fluoresce after administration. Other possible indications included intramedullary spinal ependymomas and brain metastases.

Another possible role of 5-ALA is for photodynamic therapy. ${ }^{8}$ Dr. Hadjipanayis briefly discussed this emerging area in the therapy of HGG tumors.

\subsection{5-ALA Dartmouth Experience}

Dr. David Roberts is one of the few American neurosurgical investigators with an early IND for 5-ALA and given his interest in stereotaxy. He has carefully quantified and correlated dye fluorescence with spatial location and tumor. Dr. Roberts described many practical lessons for the interpretation of fluorescent visualization with conventional neurosurgical microscopes. In an important clinical trial, Roberts et al. ${ }^{9}$ correlated tumor fluorescence with MRI contrast enhancement ( $p=$ $0.018)$ and tumor pathology $(p<0.001)$, again confirming test characteristics of $75 \%, 71 \%, 95 \%$, and $26 \%$ for 5-ALA sensitivity, specificity, PPV, and NPV, respectively. In collaboration with his engineering colleagues, Dr. Roberts has been on the forefront of spectroscopic technologies, including quantitative approaches and spectrally resolved quantitative imaging even with multiple simultaneous fluorophores such as fluorescein and 5-ALA in the same patient. ${ }^{10-14}$ Dr. Roberts emphasized the importance of the detection technology and the importance of collaboration with his engineering colleagues, with particular mention of Keith Paulsen, Pablo Valdes, and Brian Pogue.

In a side note, Dr. Roberts also described his early phase 0 experience of the ABY-029 anti-EGFR affibody in HGG patients, mostly recurrent glioblastoma. ${ }^{15,16}$ The signal-to-noise ratio was very promising at 5.7 in the first three patients. The Dartmouth group continues to expand the role of ABY-029 for multiple histologic types, which was described in more detail by Dr. Eric Henderson later in the conference.

\section{Ongoing Phase 3 Trials}

\subsection{Folate Receptor Alpha for Ovarian Cancer}

The first phase 3 clinical trial that was discussed was an ongoing randomized, multicenter, single-dose, open label study of folate receptor (FR) targeted IMI for ovarian cancer. This NIR contrast agent (OTL38) is a folate ligand attached to a NIR fluorophore (excitation $774 \mathrm{~nm}$ and emission $794 \mathrm{~nm}$ ). Prior pilot studies and a phase 2 study had already been performed, and they had shown the folate-receptor targeted contrast agent is safe..$^{17}$ In the discussion, the principal investigator, Dr. Janos Tanyi from the Perelman School of Medicine at the University of Pennsylvania, summarized the findings from the phase 2 clinical trial. In the study, 48 patients were enrolled and all patients received molecular imaging; $48 \%$ of the subject had at least one additional pathology-confirmed lesion by NIR imaging that had been missed by white light imaging alone.

A phase 3 trial is now ongoing to determine the value IMI has for patients with ovarian cancer. The primary role of surgery in ovarian cancer is surgical debulking. Thus the major value of IMI is to improve the degree of cytoreduction at the time of surgery. The primary study objective is to confirm the efficacy of IMI in detecting additional ovarian cancer lesions that are not detected by standard-practice palpation and visualization during primary surgical cytoreduction, interval debulking, or recurrent ovarian cancer surgery. In this study, the secondary endpoints are to estimate the proportion of FR-positive ovarian cancer patients in whom all lesions, without regard to evaluable lesion status, are detected by fluorescent light. Also, in this study, the safety of using this dye will be confirmed from the prior pilot and phase 1 studies.

Briefly, in the phase 3 study, Dr. Tanyi revealed that all patients are receiving a single dose of $0.025 \mathrm{mg} / \mathrm{kg}$ of OTL38 at least $1 \mathrm{~h}$ before surgery. Then during the surgery, white-light assessment is performed to identify tumor nodules. Prior to any resection, the patient is then randomized in the operating room to surgery using white-light only versus white-light and NIR. 
The surgeon is mandated to develop a surgical plan based on preoperative CT, PET and/or MRI, and white light visualization. At that point, any changes to the operation after viewing the NIR images are monitored. Patient accrual for this study is expected to take 15 months. Once again, the primary endpoint is the ability of IMI to identify additional tumors beyond what is discovered by manual palpation and visual inspection.

\subsection{Anti-CEA Receptor for Colorectal Cancer}

Following the discussion of ovarian cancer, Dr. Alex Vahrmeijer from Leiden University discussed an upcoming phase 3 clinical trial in patients with colorectal carcinoma. Based on results from the International Watch and Wait Database, they found almost $25 \%$ of patients $(n=1000)$ develop local recurrence within two years of the neoadjuvant chemoradiation therapy if they do not undergo surgical resection. ${ }^{17}$ This motivated a study to target carcinoembryonic antigen (CEA) receptor with a fluorescent dye. CEA is a glycoprotein involved in cell adhesion and is not affected by radiation therapy or chemotherapy. The molecular imaging contrast agent (SGM101 SurgiMab) is the conjugation of the chimeric monoclonal antibody SGM-Ch511 to a NIRemitting fluorochrome BM-104 (emission $700 \mathrm{~nm}$ ).

In the preliminary studies, 26 patients underwent a dose escalation study with $5,7.5$, and $10 \mathrm{mg}$ of SGM101. The primary goal was to look at the safety and pharmacokinetics of the fluorophore, as well as the accuracy of intraoperative tumor detection. Overall, IMI with SGM101 had a sensitivity of $98 \%$, specificity of $62 \%$, PPV of $81 \%$, and a negative predictive value of $94 \%$. In the study, 44 additional malignant lesions were detected with IMI after standard white-light resection. In 6/17 patients in the final dose cohort, intraoperative imaging changed treatment strategy.

Based on these results, Dr. Vahrmeijer and colleagues are initiating a phase 3 trial in the United States, Italy, Germany, and the Netherlands in 2019. The contrast agent will be used to localize primary and recurrent colorectal tumors, as well as to detect infiltrated lymph nodes, liver metastases, and peritoneal metastases. The inclusion criteria will be patients with $\mathrm{T} 4$ colon cancer, T3/T4 rectal cancer, recurrent colorectal cancer, and patients with possible peritoneal metastases. The dual endpoints will be to look at the impact of IMI in identifying additional lesions and discriminating between benign and malignant tissue. The patients $(n=300)$ will be administered the drug 3 to 5 days prior to surgery. At the onset of the operation, the surgeon will perform a white-light inspection. Then the patients will be randomized to either standard surgical procedure or NIR inspection and resection of any new suspected tissue. Data will be collected to determine how frequently there is a change in surgical plan because of IMI with SGM101. Pathology will be the gold standard.

\section{Phase 2 Trials}

\subsection{Anti-EGFR Antibody Imaging for Head and Neck Cancers}

The next discussion focused on IMI of head and neck cancers using fluorescently labeled anti-EGFR antibodies. Dr. Eben Rosenthal from Stanford University showed that 95\% of head and neck cancers express epidermal growth factor receptor (EGFR). His group has developed two different antibodies (cetuximab and panitumumab) conjugated to IRDye800 for surgical navigation during head and neck cancer surgery. IMI has two major value statements in head and neck cancer. ${ }^{18}$ First, it can provide surgical guidance for identification of cancer cells in the head and neck as well as in vivo margin assessment. Second, it can improve specimen mapping; resected specimens from the head and neck can be difficult to orient, which presents significant difficulty in locating the site of positive margins that are discovered from the specimen in the pathology suite. Thus the primary value of IMI in head and neck cancer would be in detecting margins both in vivo and ex vivo.

In his in vivo work, Rosenthal was able to show that EGFRtargeted IMI could detect satellite lesions in the oral cavity that were undetectable by white-light. One of the most fascinating data was the demonstration of NIR fluorescence that could discriminate lymph nodes containing cancer deposits from benign nodes. A third application of this technology was locating microscopic disease in the surgical wound bed after resection of the primary tumor. Interestingly, Dr. Rosenthal's ex vivo analysis provided sufficient sensitivity to discriminate tumors that had low versus high EGFR expression before final pathology. Finally, localizing tumor margins with surgical specimen mapping using NIR fluorescence was superior than the surgeon simply placing mechanical markers to orient the specimen for the pathologist.

In the discussion of IMI for head and neck cancer, Rosenthal provided his personal experience and described the importance of controlling the operating room environment for open surgery. The head and neck surgery provides unique challenges because of background noise from the mucosal surfaces and smaller incisions.

\subsection{Folate Receptor Alpha for Lung Adenocarcinoma}

Following Dr. Rosenthal's presentation, Dr. Sunil Singhal from the Perelman School of Medicine at the University of Pennsylvania discussed ongoing applications of FR-targeted imaging using OTL38 for lung adenocarcinomas. First, he presented evidence from his experience in 65 patients in pilot and phase 1 studies. ${ }^{19-21}$ First, the initial experience showed that FR-targeted IMI was successful not only for adenocarcinomas but also for ground glass opacities, a potential precursor for malignant lung adenocarcinoma, and a rapidly growing problem in the United States and Asia. ${ }^{22}$ The second finding from the pilot studies was that IMI combined with PET was superior in detecting and locating tumors for removal; in fact, IMI was better than PET for locating nodules $<1 \mathrm{~cm} .{ }^{23}$ Finally, despite theoretical specificity for lung adenocarcinomas, lung squamous cell carcinomas were also sensitive to OTL38 because of off-target effects on FR-beta positive tumor-associated macrophages. ${ }^{24}$

Dr. Singhal also presented the preliminary findings from the phase 2 study. In that study, three endpoints were defined as "clinically significant events." The first was the number of times the surgeon performed surgery for a single lesion and then simultaneously discovered additional cancer outside the intended operation. The second endpoint was the number of incidences that the surgeon felt the resection was a negativemargin operation, yet IMI discovered positive margins. The final endpoint was the number of patients in whom the surgeon could not locate the tumor during minimally invasive surgery, yet IMI was able to pinpoint the location for a lung wedge biopsy. The results were promising and the phase 3 study was outlined based on these three significant events. 


\subsection{Cathepsin-Activated Imaging for Postbreast Lumpectomy Margins}

Dr. Barbara Smith from the Harvard University School of Medicine discussed her phase 2 experience with a protease-activated system for real-time breast cancer lumpectomy margin assessments. The chief problem she tackled was the presence of positive margins during lumpectomy that would lead to repeat surgeries. Her group is studying a cathepsin and MMP-activated fluorophore (LUM015) that is injected intravenously 2 to $6 \mathrm{~h}$ prior to surgery. After standard lumpectomy, the cavity is imaged with a hand-held imaging device that detects activated LUM015 fluorescence $(670 \mathrm{~nm})$. She explained that an area with a diameter of $2.6 \mathrm{~cm}$ could be imaged per second. In their 60-patient trial, they examined LUM015 safety, the rate of true and false positive detection with fluorescence imaging, and refined a threshold algorithm.

Dr. Smith's study had a mean tumor size of $1.2 \mathrm{~cm}$ with $60 \%$ of the patients having invasive ductal and/or ductal carcinoma in situ. Both abnormal (prior biopsy sites and fibrocystic changes) and cancerous tissues were fluorescent. Eight patients had positive final margins by histopathology and had intraoperative cavity margin imaging. Two of those patients had a positive cavity reading and a wider margin was taken, thereby saving the patients from a second surgery. In 3 of 4 cases, the IMI demonstrated positive cavity readings but a wider margin was not deemed necessary by the surgeon.

IMI with LUM015 had 100\% sensitivity for tumors $<2 \mathrm{~mm}$ from the margin and had excellent sensitivity (84\%) even for tumors with intermediate margins. In a subgroup analysis, Dr. Smith showed that IMI was not affected by breast density or menopausal status. Thus using IMI for margin status in breast lumpectomies shows promise and will be explored in future studies to investigate the impact of neoadjuvant therapy, to refine the IMI algorithms for different histological subtypes, and to assess the degree of residual tumor after negative IMI readings.

\section{Phase 1 Trials and Pilot Studies}

After the morning discussions of phase 2 and phase 3 trials and their endpoints, the next topic was presentations on new up and coming NIR contrast agents that are being tested for safety and toxicity in preparation for efficacy trials. These presentations provided a window into future developments of IMI.

\subsection{Anti-EGFR Affibody NIR Imaging of Soft Tissue Sarcomas}

Dr. Eric Henderson of Dartmouth College presented his findings using ABY-029, an anti-EGFR affibody linked to IRDye 800cw, in soft-tissue sarcomas. The advantage of affibodies is that they are $\sim 5 \%$ of the mass of antibodies, allowing rapid clearance, less toxicity, and less trapping in the reticuloendothelial system of the body. Over 10 studies have shown that the average frequency of EGFR expression and amplification in sarcomas was $60 \%$, thus making this a reasonable target for IMI. In his preclinical murine studies, Dr. Henderson demonstrated that tumors could be imaged through up to $3 \mathrm{~cm}$ of tissue, a depth that sarcoma surgeons would routinely experience in surgery. ${ }^{25}$ Furthermore, in sophistical phantom models, his group showed that a signal-to-background ratio of 2:1 was sufficient in the tissues typical in extremities to be able to identify tumors.
In pharmacokinetic studies, a signal-to-background ratio of 3.5 could be consistently achieved.

His group has successfully entered into a human study and performed a phase 0 microdose study with a goal accrual of 18 patients. Even at the lowest dose, they achieved signal-tobackground ratios from 3- to 30-fold compared to surrounding tissues. The signal intensity was directly correlated to the level of EGFR expression in the cancers. The tumor periphery fluoresced most strongly, which is promising because this is the area that will be most relevant to the surgeons.

\subsection{TumorGlow ${ }^{T M}$ for Adult Brain Tumors}

Dr. John Y. K. Lee described a novel application of an already FDA-approved dye, indocyanine green (ICG). Although ICG has been used as a vascular contrast agent for decades, its use as a tumor contrast agent has only recently been exploited by surgeons at Penn and has been termed Second-Window ICG (SWIG) or TumorGlow ${ }^{\mathrm{TM}}$. TumorGlow ${ }^{\mathrm{TM}}$ functions by administering ICG at much higher doses (2.5 to $5 \mathrm{mg} / \mathrm{kg}$ ) than used in vascular angiography $(0.35 \mathrm{mg} / \mathrm{kg})$, and by waiting $24 \mathrm{~h}$ ("second window") for the intravascular ICG to clear out of the system and out of background. ${ }^{26}$ This approach has allowed neurosurgeons to visualize fluorescence confined to the neoplastic tissue of HGG, meningiomas, metastases, and pituitary adenomas with surprisingly high signal-to-background ratios. ${ }^{27-32}$ Indeed, the visualization of abnormal tissue is very similar to principles of gadolinium contrast-enhancement in MRI, which relies on passive diffusion of contrast agent into abnormal tumor tissue with permeable vascular endothelium. Since gadolinium enhancement is the very basis of the definition of gross total resection or partial resection of glioma in surgical outcome studies for glioblastoma, SWIG TumorGlow ${ }^{\mathrm{TM}}$ has the potential to help neurosurgeons increase the extent of resection in HGG resections.

Dr. John Lee then presented unpublished data in 55 HGG, both recurrent and primary, showing sensitivity, specificity, PPV, NPV of $96 \%, 54 \%, 95 \%, 58 \%$, respectively, for this SWIG TumorGlow ${ }^{\mathrm{TM}}$ technique. Based on the FDA approval of 5-ALA using PPV alone, SWIG shows promise as an alternative to 5-ALA, and indeed it may be more sensitive than 5-ALA, especially in metastases and other contrast-enhancing brain tumors, such as meningioma, juvenile pilocytic astrocytoma, ependymoma, and others.

\subsection{TumorPaint ${ }^{T M}$ for Pediatric Brain Tumors}

In children, the most common solid tumor is brain tumors, and for the vast majority of histologic types, the extent of resection determines long-term survival. With this background, Dr. Amy Lee from the University of Washington School of Medicine presented the pediatric experience of a novel drug, toluzeristide (BLZ-100 or TumorPaint ${ }^{\mathrm{TM}}$ ) in conjunction with two novel imaging camera systems. Tozulerstide is a chlorotoxin-based, tumor-targeting peptide conjugated to ICG. Dr. Amy Lee presented a dose escalation study (three patients; each dose from 1.7 to $17.3 \mathrm{mg} / \mathrm{m}^{2}$ ) demonstrating higher fluorescent intensity with increasing doses. Of 28 patients enrolled in their pilot study, 23 patients $(82 \%)$ with a wide histologic range of tumors demonstrated fluorescence, including medulloblastoma, ependymoma, pleomorphic xanthoastrocytoma, juvenile pilocytic astrocytoma, atypical teratoid rhabdoid tumor, and HGG. Curiously, though, one medulloblastoma, one HGG, and one 
nonenhancing oligodendroglioma did not fluoresce after toluzeristide administration.

Dr. Amy Lee also described two imaging systems that she used to visualize NIR fluorescence: the SIRIS exoscope system and CANVAS exoscope system, both of which attach to the front of Zeiss Pentero neurosurgical microscopes. These systems were designed to overcome the limitations of the conventional neurosurgical microscopes in detection of ICG signal.

Toluzeristide is moving forward with a multisite clinical trial in the United States within the Pediatric Neuro-Oncology Consortium. Clinical design endpoints were not elaborated in this presentation.

\subsection{Fluorescein for Gliomas}

Dr. Jeffrey Bruce described the role of fluorescein in HGG resections. Fluorescein is only a fraction of the cost of 5-ALA, is nontoxic, and has been FDA-approved for decades. Fluorescein is injected upon induction of anesthesia, thus allowing it to circulate for $\sim 1$ or $2 \mathrm{~h}$ prior to surgical visualization using conventional neurosurgical microscopes fitted with a Yellow560 filter. Empiric evidence points to fluorescein's value as a means of identifying gadolinium contrast-enhancing tissue. Using fluorescein in a single-center, 32-patient cohort study using historical controls, Dr. Bruce demonstrated a GTR rate of $93.1 \%$ versus $77.3 \%$ using fluorescein. ${ }^{33}$ In nonenhancing gliomas, Dr. Bruce has also found some potential utility of fluorescein.

One advantage of fluorescein as compared to 5-ALA and NIR fluorophores is the brightness of the dye. Dr. Bruce explained that it can be difficult to see much anatomical detail when using 5-ALA filters alone, and indeed Dr. Stummer did comment that the neurosurgical microscope filters for 5-ALA were modified to allow some blue excitation light to pass through the emission filter as many surgeons complained that they could not operate with red filtered light only. In contrast, fluorescein's excitation and emission are in a color range that more closely mimics the natural brain, allowing the surgeon to "see" surrounding tissue as well as red blood vessels without difficulty when using fluorescein-specific filters.

\subsection{TumorGlow ${ }^{T M}$ for Head and Neck Carcinomas}

Dr. Jason Newman from the University of Pennsylvania discussed applications of TumorGlow ${ }^{\mathrm{TM}}$ to squamous cell carcinomas and salivary gland carcinomas of the head and neck. ${ }^{34} \mathrm{He}$ presented his 33-patient series and his primary finding was the value of this technology using transoral robotic surgery (TORS). In his pilot study, IMI was a powerful adjunct to help surgeons make up for the loss of haptics in TORS compared to open surgery.

Results were preliminary but showed that the TumorGlow ${ }^{\mathrm{TM}}$ approach was safe and feasible. The dye localized to the tumor and then seemed to passively drain to the draining lymph nodes, providing a "sentinel lymph node" result. Future studies will begin to explore possible endpoints surrounding the sensitivity and specificity of identifying relevant lymph nodes.

\section{Conclusions}

This conference was useful in bringing together investigators steeped in clinical trials in IMI across a wide range of surgical specialties. In contrast to prior meetings, which have primarily focused on scientists and novel imaging agents and techniques, this course was designed to congregate surgical investigators with practical human experience in diverse cancer types. Several themes emerged.

All cancer surgeons rely on pathological confirmation of results. Surgery is part of a continuum that starts with preoperative imaging studies (e.g., CAT scan and MRI scan), followed by tumor resection, and then completed with pathological validation. IMI fills a niche between preoperative imaging and pathology. This optical imaging modality can even augment preoperative imaging studies at the time of surgery. ${ }^{23,29}$ Interestingly, because of rapid growth and change in molecular diagnosis of cancer, at times even the gold standard definition of tumor may not be the same today as it will be in five years. Hence, definitions of sensitivity/specificity were challenged by some of the investigators, but the universal truth remains that identification of pathologic tissue remains paramount for IMI.

Since each cancer has unique methods of invasiveness and because organs of tumor cell origin are so varied in function, the seminar revealed important and unique challenges. This was most evident in the endpoints that each specialty was studying in clinical trials.

A major common goal in cancer surgery is identifying synchronous and metachronous lesions that may exist at the time of surgery. In the phase 3 colon cancer trial and the phase 2 lung cancer trial, both studies shared this common endpoint. The fact that these studies have found IMI helpful for intraoperative detection of additional disease at the time of surgery not previously identified on preoperative noninvasive imaging speaks to the ability of intraoperative optical imaging to improve upon preoperative imaging. Indeed, most preoperative imaging outside of the brain requires ionizing radiation and has a 5-mm to $1-\mathrm{cm}$ size limitation. This has not been shown to be the case for optical molecular imaging as nodules as small as $<5 \mathrm{~mm}$ can be identified. Since the surgeon has direct access to the region where the tumor is located, IMI can visualize small quantities of disease using the surgeon's eyes to look for fluorescing cancer cells.

As important as identification of additional deposits of tumor may be for colon cancer and lung cancer, this is not exactly the same problem for cancers of the brain. In HGG surgery, identification of synchronous and metachronous lesions is less important because of the high spatial fidelity of magnetic resonance imaging, which allows detection of 1-mm brain metastases. Thus the advantage of IMI in brain surgery is margin detection. Given the challenges of systematic techniques for peritumoral biopsy in highly functional organs of delicate organization like the brain, surgeon investigators have been able to convince the FDA that the high PPV of IMI using 5-ALA is helpful. Hence, pooled sensitivity and specificity values of $<80 \%$ were accepted by the FDA for surgical imaging label in HGG.

Other cancer types that benefit from margin assessment are head and neck and breast cancers. This was best exemplified by the head and neck cancer trials using the anti-EGFR antibodies and breast cancer trials using cathepsin-activated enzymatic dyes. The ability to examine margins in vivo and ex vivo each has advantages. In vivo, imaging of tumor margins allows for extraordinary sensitivity for the surgeons' blade. Not only the head and neck and breast cancer surgeons, but also the neurosurgeons echoed that 5-ALA and TumorGlow ${ }^{\mathrm{TM}}$ offered similar benefits. Both the neurosurgeons and otorhinolaryngologists felt that the ability to use IMI in the wound bed after the tumor has been removed was an important adjunct. Similar data have recently come out for IMI during sarcoma and thymoma 
surgeries. ${ }^{35,36}$ Second, ex vivo imaging of tumor margins allowed for accurate "specimen mapping" on the back table, which permitted better orientation of en bloc resections after being removed from the patients.

Another common theme that arose in the seminar was the concept of "optical biopsy," that is, using IMI to discriminate benign from malignant tissue. ${ }^{37}$ In the phase 3 colorectal cancer trial, this was one of the two endpoints. The targeted CEA tracer would distinguish tissue that looks abnormal in the liver, pelvis, omentum, and lymph nodes. Similarly, in the phase 2 lung cancer trial, a secondary endpoint was to examine if IMI could discriminate benign and premalignant tumors from malignant nodules. In this era of molecular targeting, it appears that this optical biopsy will be a major value of the new and upcoming dyes. The anti-EGFR targeted probe in the studies at Stanford University also suggested the same value, although no data was specifically presented on this endpoint. The ability to conjugate fluorophores to cellular targets provides a level of specificity that has not existed with nonreceptor specific dyes, such as 5-ALA.

Many tumors are not completely resectable. Thus the goals of surgery are often cytoreduction to eliminate as much as disease as possible in order to allow chemotherapy and/or radiation therapy to focus on small quantities of residual disease. Examples of this include ovarian cancer surgery, mesothelioma surgery, and pulmonary metastectomy (e.g., sarcoma, colorectal, and germ cell). Therefore, it is not surprising that the primary endpoint in the ongoing phase 3 ovarian cancer clinical trial is tumor cytoreduction. None of the other surgeons discussed this as a primary endpoint. Although not presented at this conference, ongoing work in utilizing IMI for pulmonary metastectomy is underway. ${ }^{38,39}$

In summary, IMI has emerged over the last decade and will likely alter the face of cancer surgery. 5-ALA has led the way in glioma surgery, but others are certain to follow. There are $\sim 4$ to 5 common themes that all cancer surgeons share and the next decade will surely prove the clinical value of this approach.

\section{Disclosures}

The following authors have disclosed affiliations with the following industry partners. John Y. K. Lee MD: VisionSense; Walter Stummer MD PhD: Medac, NxDC, Zeiss Meditech; Eben Rosenthal MD: Intuitive; David W. Roberts MD: Alcyone, CarThera, ODS Medical, Insight Surgical Technologies; Constantinos G. Hadjipanayis, MD PhD: Meditech, Synaptive, Nx Development Corporation; Jason G. Newman ND: Medtronic, Castle Biosciences, VisionSense, JustRight Surgical. No conflicts of interest, financial or otherwise, are declared by the other authors.

\section{References}

1. W. Stummer et al., "Fluorescence-guided surgery with 5-aminolevulinic acid for resection of malignant glioma: a randomised controlled multicentre phase III trial," Lancet Oncol. 7, 392-401 (2006).

2. C. G. Hadjipanayis and W. Stummer, "5-ALA and FDA approval for glioma surgery," J. Neurooncol. 141, 479-486 (2019).

3. J. S. Cordova et al., "Quantitative tumor segmentation for evaluation of extent of glioblastoma resection to facilitate multisite clinical trials," Transl. Oncol. 7, 40-47 (2014).

4. J. S. Cordova et al., "Semi-automated volumetric and morphological assessment of glioblastoma resection with fluorescence-guided surgery," Mol. Imaging Biol. 18, 454-462 (2016).
5. J. S. Cordova et al., "Whole-brain spectroscopic MRI biomarkers identify infiltrating margins in glioblastoma patients," Neuro-Oncol. 18, 1180-1189 (2016).

6. R. Díez Valle, C. G. Hadjipanayis, and W. Stummer, "Established and emerging uses of 5-ALA in the brain: an overview," J. Neurooncol. 141, 487-494 (2019)

7. G. Widhalm et al., "5-Aminolevulinic acid induced fluorescence is a powerful intraoperative marker for precise histopathological grading of gliomas with non-significant contrast-enhancement," PLoS One 8, e76988 (2013).

8. K. Mahmoudi et al., "5-aminolevulinic acid photodynamic therapy for the treatment of high-grade gliomas," J. Neurooncol. 141, 595-607 (2019).

9. D. W. Roberts et al., "Coregistered fluorescence-enhanced tumor resection of malignant glioma: relationships between $\delta$-aminolevulinic acidinduced protoporphyrin IX fluorescence, magnetic resonance imaging enhancement, and neuropathological parameters," J. Neurosurg. 114, 595-603 (2011).

10. K. Bekelis et al., "Quantitative and qualitative 5-aminolevulinic acidinduced protoporphyrin IX fluorescence in skull base meningiomas," Neurosurg. Focus 30, E8 (2011).

11. P. A. Valdés et al., "Quantitative fluorescence using 5-aminolevulinic acid-induced protoporphyrin IX biomarker as a surgical adjunct in low-grade glioma surgery," J. Neurosurg. 123, 771-780 (2015).

12. M. Sibai et al., "Quantitative subsurface spatial frequency-domain fluorescence imaging for enhanced glioma resection," J. Biophotonics 12, e201800271 (2019).

13. D. W. Roberts et al., "Red-light excitation of protoporphyrin IX fluorescence for subsurface tumor detection," J. Neurosurg. 128, 16901697 (2018).

14. P. A. Valdés et al., "Quantitative, spectrally-resolved intraoperative fluorescence imaging," Sci. Rep. 2, 798 (2012).

15. J. T. Elliott et al., "Simultaneous in vivo fluorescent markers for perfusion, protoporphyrin metabolism, and EGFR expression for optically guided identification of orthotopic glioma," Clin. Cancer Res. 23, 2203-2212 (2017).

16. A. L. R. de Souza et al., "Fluorescent affibody molecule administered in vivo at a microdose level labels EGFR expressing glioma tumor regions," Mol. Imaging Biol. 19, 41-48 (2017).

17. G. M. van Dam et al., "Intraoperative tumor-specific fluorescence imaging in ovarian cancer by folate receptor- $\alpha$ targeting: first in-human results," Nat. Med. 17, 1315-1319 (2011).

18. R. W. Gao et al., "Determination of tumor margins with surgical specimen mapping using near-infrared fluorescence," Cancer Res. 78, 51445154 (2018).

19. J. D. Predina et al., "A phase I clinical trial of targeted intraoperative molecular imaging for pulmonary adenocarcinomas," Ann. Thorac. Surg. 105, 901-908 (2018).

20. J. D. Predina et al., "Standardization and optimization of intraoperative molecular imaging for identifying primary pulmonary adenocarcinomas," Mol. Imaging Biol. 20, 131-138 (2018).

21. J. D. Predina et al., "Identification of a folate receptor-targeted nearinfrared molecular contrast agent to localize pulmonary adenocarcinomas," Mol. Ther. 26, 390-403 (2018).

22. J. D. Predina et al., "Localization of pulmonary ground-glass opacities with folate receptor-targeted intraoperative molecular imaging," J. Thorac. Oncol. 13, 1028-1036 (2018).

23. J. D. Predina et al., "Intraoperative molecular imaging combined with positron emission tomography improves surgical management of peripheral malignant pulmonary nodules," Ann. Surg. 266, 479-488 (2017).

24. J. D. Predina et al., "An open label trial of folate receptor-targeted intraoperative molecular imaging to localize pulmonary squamous cell carcinomas," Oncotarget 9, 13517-13529 (2018).

25. K. S. Samkoe et al., "Preclinical imaging of epidermal growth factor receptor with ABY-029 in soft-tissue sarcoma for fluorescence-guided surgery and tumor detection," J. Surg. Oncol. 119, 1077-1086 (2019).

26. R. Zeh et al., "The second window ICG technique demonstrates a broad plateau period for near infrared fluorescence tumor contrast in glioblastoma," PLoS One 12, e0182034 (2017).

27. J. W. Jeon et al., "Near-infrared optical contrast of skull base tumors during endoscopic endonasal surgery," Oper. Neurosurg. 17, 32-42 (2019). 
28. J. Y. K. Lee et al., "Near-infrared fluorescent image-guided surgery for intracranial meningioma," J. Neurosurg. 128, 380-390 (2018).

29. J. Y. K. Lee et al., "Intraoperative near-infrared optical imaging can localize gadolinium-enhancing gliomas during surgery," Neurosurgery 79(6), 856-871 (2016).

30. S. S. Cho, R. Salinas, and J. Y. K. Lee, "Indocyanine-green for fluorescence-guided surgery of brain tumors: evidence, techniques, and practical experience," Front. Surg. 6, 11 (2019).

31. J. Y. K. Lee et al., "Intraoperative near-infrared optical contrast can localize brain metastases," World Neurosurg. 106, 120-130 (2017).

32. S. S. Cho et al., "Intraoperative near-infrared imaging with receptorspecific versus passive delivery of fluorescent agents in pituitary adenomas," J. Neurosurg. 1, 1:1-11 (2018).

33. J. A. Neira et al., "Aggressive resection at the infiltrative margins of glioblastoma facilitated by intraoperative fluorescein guidance," J. Neurosurg. 127, 111-122 (2017).

34. V. C. Stubbs et al., "Intraoperative imaging with second window indocyanine green for head and neck lesions and regional metastasis," Otolaryngol. Neck Surg. 161(3), 539-542 (2019).

35. J. J. Keating et al., "Intraoperative imaging identifies thymoma margins following neoadjuvant chemotherapy," Oncotarget 7, 3059-3067 (2016).

36. D. Holt et al., "Intraoperative near-infrared fluorescence imaging and spectroscopy identifies residual tumor cells in wounds," J. Biomed. Opt. 20, 076002 (2015).

37. G. T. Kennedy et al., "The optical biopsy," Ann. Surg. 262, 602-609 (2015).

38. A. D. Newton et al., "Surgical management of early-stage esophageal adenocarcinoma based on lymph node metastasis risk," Ann. Surg. Oncol. 25, 318-325 (2018).

39. J. Keating et al., "Near-infrared intraoperative molecular imaging can locate metastases to the lung," Ann. Thorac. Surg. 103, 390-398 (2017).

John Y. K. Lee is an associate professor of neurological surgery and otorhinolaryngology at Pennsylvania Hospital of the University of Pennsylvania. As $\mathrm{PI}$ or co-investigator on several university grants, he has laid the groundwork for near-infrared, fluorescent tumor resection in open brain surgery (gliomas, meningiomas, and metastasis) as well as in endoscopic brain surgery (pituitary adenomas and craniopharyngiomas) using multiple near-infrared fluorophores. He also serves as the co-director of the Penn Center for Precision Surgery, which uniquely positions him to translate his research in fluorescent brain tumor imaging directly to patients with deadly brain tumors.

Steve S. Cho is a fourth-year medical student at the University of Pennsylvania and has been working with Dr. John Lee since early 2016 on various fluorescence-guided neurosurgery projects using targeted and nontargeted fluorophores. He hopes to start his neurosurgery residency in 2020 .

Walter Stummer is the chairman of neurosurgery at the University of Münster, Germany. He was the first neurosurgeon to recognize 5-ALA as a potential agent for fluorescence-guided neurosurgery in patients with high-grade gliomas. His landmark randomized clinical trial was a key in getting 5-ALA approved in Europe and in the United States.

Janos L. Tanyi is an assistant professor of obstetrics and gynecology at the Hospital of the University of Pennsylvania. He is one of the coinvestigators in a clinical trial of OTL38, a folate analog near-infrared fluorophore, in patients with ovarian carcinoma.

Alexander L. Vahrmeijer is a surgical oncologist at Leiden University Medical Center, the Netherlands. He closely collaborates with Professor Frangioni who developed the FLARE and mini-FLARE NIR fluorescence imaging systems, which are both used in clinical trials at the LUMC in more than 300 patients. His current research focuses on the introduction of image-guided surgery using clinically available probes and on the clinical introduction of novel probes, like ZW800, in collaboration with Professor Frangioni. Together with Professor van de Velde and Professor Frangioni, he aims at disseminating this technique to collaborating hospitals all over the world.

Eben Rosenthal serves as the Ann and John Doerr Medical Director of the Stanford Cancer Center. He oversees the clinical delivery of cancer care for Stanford throughout the Bay Area. He leads a multidisciplinary team to perform translational studies including multiple first-in-human trials. He has more than 200 publications and has been continuously funded by the $\mathrm{NIH}$ for 20 years. He is clinically active in head and neck cancer surgery and microvascular reconstruction.

Barbara Smith received her undergraduate training from Massachusetts Institute of Technology, her MD from Harvard Medical School's Harvard/MIT Division of Health Sciences and Technology, and her PhD from the Graduate School of Arts and Sciences at Harvard University. She is the director of the Breast Program and co-director of the Women's Cancers Program at Massachusetts General Hospital. She is an associate professor of surgery at Harvard Medical School and at the MGH. Her research activities have focused on reducing the extent of surgery and radiation required to treat breast cancer, diagnosis and treatment issues in young women with breast cancer, and three-dimensional breast anatomy as it relates to breast surgery and pathologic analysis of specimens.

Eric Henderson is an associate professor of orthopaedic surgery and medical director of the Sarcoma and Connective Tissue Oncology Program at Dartmouth-Hitchcock Medical Center. His research goals are to enhance the safety and effectiveness of surgery by advancing optical and imaging-based surgical navigation technologies that will aid in the identification of critical tissues, clearing the path to autonomous robotic surgery.

David W. Roberts is a professor of neurosurgery and neurology at Geisel School of Medicine of Dartmouth University. His contributions to fluorescence-guided neurosurgery include developing innovative ways to visualize 5-ALA fluorescence.

Amy Lee earned her MD from the University of Texas Health Science Center, San Antonio. She is a neurosurgeon at Seattle Children's Hospital and an associate professor of neurological surgery at the University of Washington. She went on to complete a seven-year neurological surgery residency at Washington University School of Medicine in St. Louis, Missouri, and Barnes-Jewish Hospital. She then went on to complete her pediatric neurosurgery fellowship at St. Louis Children's Hospital. Her clinical interests include craniofacial anomalies, spinal dysraphism, hydrocephalus, and brain tumors. Her research interests include magnetic resonance imaging techniques, diffusion tensor imaging in traumatic brain injury and spinal cord injury.

Constantinos G. Hadjipanayis is a professor of neurosurgery and oncological sciences at Icahn School of Medicine at Mount Sinai. He serves as a chair of neurosurgery at Mount Sinai Union Square/ Beth Israel and a director of Neurosurgical Oncology for the Mount Sinai Health System. He is a director of the Mount Sinai Brain Tumor Nanotechnology Laboratory where he studies innovative brain tumor therapeutics. He has led the development of 5-ALA (Gleolan) and fluorescence-guided surgery in the United States.

Jeffrey $\mathbf{N}$. Bruce is a professor of neurosurgery at Columbia University Medical Center. He leads a multidisciplinary effort as codirector of the Brain Tumor Center and a director of skull base surgery specializing in the surgical treatment of gliomas, meningiomas, pituitary tumors, skull base tumors, craniopharyngiomas, and pineal tumors. His surgical expertise includes extensive experience with minimally invasive techniques and stereotactic surgery as well as surgical alternatives such as radiosurgery. As co-director of the Brain Tumor Center, he leads an $\mathrm{NIH}$-funded translational brain tumor research effort with interests in immunotherapy and drug delivery systems.

Jason G. Newman is an associate professor of otorhinolaryngology at the Hospital of the University of Pennsylvania. He is now exploring the use of near-infrared fluorescence in head-and-neck surgeries.

Sunil Singhal is the William Maul Measey Associate Professor in the Department of Surgery of the Perelman School of Medicine at the University of Pennsylvania, and he is the vice chair for translational research in the Department of Surgery. He is an attending surgeon at the Hospital of the University of Pennsylvania, the director of the Center for Precision Surgery at the Abramson Cancer Center, and the director of the Thoracic Surgery Research Laboratory. 